\title{
College Collaboration Portal with Training and Placement
}

\author{
ShilpaHadkar,SnehalBaing,TruptiHarer,SonamWankhade, K.T.V.Reddy \\ I. T. Department, PadmabhushanVasantdadaPatilPratishthan's College Of Engineering. Sion (East), Mumbai- \\ 400022
}

\begin{abstract}
Training and placement is the crucial part of any educational institute in which most of the work is being done manually. The aim of or project is Automation of Training and Placement until that will include minimum manual work and maximum optimization, abstraction security. This is web application which will help student as well as the administration authority to carry out each activity in this department.
\end{abstract}

Keywords-Automation, Optimization, Security, Task, activity, interface, Training information, software and placement.

\subsection{Training and Placement cell}

\section{Introduction}

The software for the training and placement cell of a college is a vital need for the students and the institute management for the purpose of proper placement and training of the aspiring students of the institute. It helps the students to provide their profiles to the training and placement cell of the institute, update their respective profiles (with their gradual approach towards course end), get to know about the companies coming for the on campus/off campus/pool/group pool categories of campus interviews. Under this system all the students of the ongoing (current) batches of various streams can register their profiles.

The training and placement cell ( $t / p$ cell) remains in continuous touch with all the students of the institute time-to-time .This dedicated software for the institute's $\mathrm{t} / \mathrm{p}$ cell can be expanded to the various machines in the institute and can be accessed by the students as well as by the administrator (tpo in charge).The system remains connected with the profile databases of the various students to continuously monitor the variety of scopes available for the students' placement.

\subsection{Overview}

This project is mainly intended for automating this procedure that can help the people who belong to the T\&P cell by saving their time, based on this basic operation

Actually their activity is under two steps the first one is, to maintain the list of students and their credit records. The second job is to maintain the company details and based on the company requirements, need to select the students and make the list of students branch wise, which is more complex task, and here informing is through notice boards, whereas this is also a bit old fashioned task, which can be automated in our proposed system by sending mails to the respective candidates

\subsection{Existing System}

\section{Existing system and Proposed System}

The existing Placement cell was not so continent because of maximum manual work. All the work that is done until now is done by human intervention. As all the work is done manually, there were maximum chances of errors. The interface of student and administer is maximum.

Due to the above problems every procedure becomes time consuming. The records were stored in modified access sheets hence sorting problem. The files were not stored hierarchical format hence searching problems.

Also the updating was very difficult and ambiguous. The duplication of records was usual hence data redundancy. The students were not being made aware of the $\mathrm{TnP}$ activity hence there might have been loss of opportunities. The Access sheets were less optimized e.g. suppose we want students having 2 ATKT then the student with 0,1,2 ATKT were select whereas required result is only of 2ATKT.

The system now could not take acknowledgement from the students attending to particular event hence lots of confusion at the last moment.

There were fewer interfaces between student and TnP department. There was no record kept of the past students. There was less communication between past or present student with the TnP department. 


\subsection{Design Goals}

In this paper, we try to solve the problem of student and the staff for using the training and placement details by adapting the automation of this cell. So goal of our project are:

1) Reduces the work pressure of the T/P cell staffs

2) Provides the statistics of students placed

3) Informing students about the events going to take

4) Easy retrieval of student data

\subsection{Proposed System}

Traditionally the job of registration was done manually by passing the registration form to the students. But this was too much time consuming and also erroneous. So the major need was for the automation for registration by online registration by students themselves.

The files in which the data is stored is stored in Access file sheets that too separately for each class of department, so the files could be accessed by any one accessing the computer. These files may be 'confidential'. So there is a special need for security.

The Calculations done until now was done manually and fed into the access sheets. So the need arises for the automatic calculation of average/aggregate.

The modified access sheets were not that efficient as, when we want to select students having 2 ATKT then the student with 0,1 , and 2 ATKT were select whereas required result is only of 2ATKT.

As mentioned earlier the data was stored separately for classes of each department the problem of searching was time consuming and as well the duplication could occur. So there is a need for a centralized hierarchical structure.

The only method for notification until now is by notice board which is not reliable. To countermeasure this problem the notification can be send by E-mail and mobile sms.

The alumni data is insufficient and is out of reach of the students. So this is proper method to employed to store the alumni data.

\section{Figures}

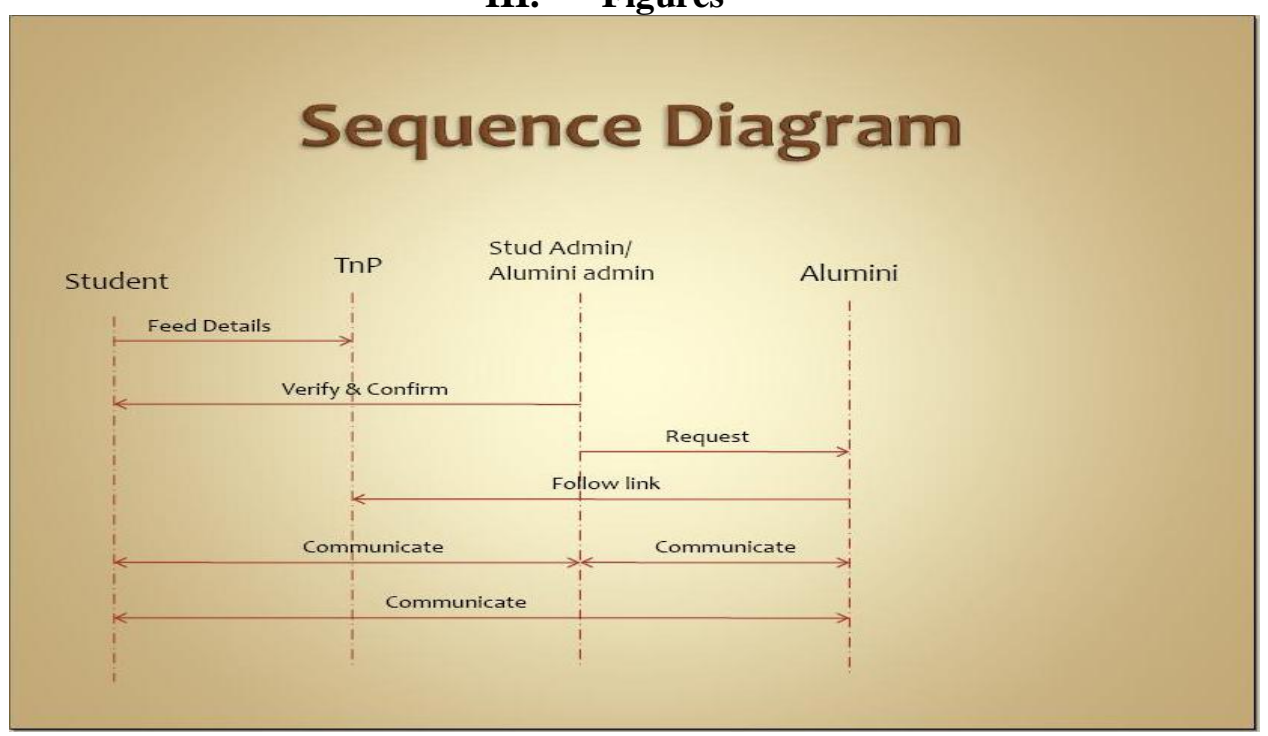

Fig 1: sequence diagram

\section{Conclusion}

To overcome all the limitations we are developing a web application for the automation of Training and Placement department. This web application will perform various tasks for student administrative authority.

This project helps a better student support and time saving factor. It also supports better student's queries and would also provide better reports and information to the management for smooth running. There is no chance of human error, as the various reports will be printed without any human intervention. The various types of reports will be available quickly without any effort.

The project named Training and Placement is application created in ASP.net. The project has been developed on the basis of "Placement Cell" being presently used in our college for storing and retrieving the information of students and companies who are registered in placement cell. The Placement Cell maintains a 
large database of students wherein all the information of student including the personal records and the academic performance in terms of the marks is stored and company information includingresume of company and facilities it provide. The software retrieves this data and displays as per the user requirement.

\section{REFERENCE}

[1] R. Ostrovsky, "Software protection and simulations on oblivious rams," Ph.D dissertation, Massachusetts Institute of Technology, 1992.

[2] V. Levenshtein, "Binary codes capable of correcting spurious insertions and deletions of ones," Problems of Information Transmission, vol. 1,no.1, pp. 8-17, 1965

[3] Tech terms: what every telecommunications and digital media person should know By Jeff Rutenbeck, Jeffrey Blaine

[4] Microsoft Silverlight 4 Business Application Development: Beginners Guide By Cameron Albert, Frank LaVigne, Packt Publishing

[5] Beginning ASP.NET 3.5: In C\# and VB (Programmer to Programmer) By ImarSpaanjaars, Wrox

[6] SQL Programming Joes 2 Pros: Programming \& Development for Microsoft SQL Server 2008 By Rick A. Morelan

[7] Kimball's Toolkit Classics: Data Warehouse Toolkit, 2ndEd; Data Warehouse Lifecycle Toolkit, 2nd Ed; Data Warehouse ETL Toolkit

[8] The Microsoft Data Warehouse Toolkit, 2nd Edition: With SQL Server 2008 R2 and the Microsoft Business Intelligence Toolset 